\title{
Spectrum Allocation for Decentralized Transmission Strategies: Properties of Nash Equilibria
}

\author{
Peter von Wrycza, ${ }^{1}$ M. R. Bhavani Shankar, ${ }^{1}$ Mats Bengtsson, ${ }^{1}$ \\ and Björn Ottersten (EURASIP Member) $)^{1,2}$ \\ ${ }^{1}$ Department of Electrical Engineering, ACCESS Linnaeus Centre, Signal Processing Laboratory,
Royal Institute of Technology (KTH), SE-100 44 Stockholm, Sweden
${ }^{2}$ Interdisciplinary Centre for Security, Reliability, and Trust, University of Luxembourg, Luxembourg 1511, Luxembourg
}

Correspondence should be addressed to Peter von Wrycza, peter.von.wrycza@ee.kth.se

Received 1 October 2008; Accepted 4 March 2009

Recommended by Holger Boche

\begin{abstract}
The interaction of two transmit-receive pairs coexisting in the same area and communicating using the same portion of the spectrum is analyzed from a game theoretic perspective. Each pair utilizes a decentralized iterative water-filling scheme to greedily maximize the individual rate. We study the dynamics of such a game and find properties of the resulting Nash equilibria. The region of achievable operating points is characterized for both low- and high-interference systems, and the dependence on the various system parameters is explicitly shown. We derive the region of possible signal space partitioning for the iterative waterfilling scheme and show how the individual utility functions can be modified to alter its range. Utilizing global system knowledge, we design a modified game encouraging better operating points in terms of sum rate compared to those obtained using the iterative water-filling algorithm and show how such a game can be imitated in a decentralized noncooperative setting. Although we restrict the analysis to a two player game, analogous concepts can be used to design decentralized algorithms for scenarios with more players. The performance of the modified decentralized game is evaluated and compared to the iterative water-filling algorithm by numerical simulations.
\end{abstract}

Copyright (C) 2009 Peter von Wrycza et al. This is an open access article distributed under the Creative Commons Attribution License, which permits unrestricted use, distribution, and reproduction in any medium, provided the original work is properly cited.

\section{Introduction}

Over the last few years, many theoretical connections have been established between problems arising in wireless communications and those in the field of game theory [1]. One such instance is when several coexisting links consisting of transmit-receive pairs compete with an objective of maximizing their individual data rates while treating the interference as Gaussian noise [2]. Due to the wireless communication channel, the received signal at each receiver is interfered by all transmitters, and the performance of the transmission strategies is, therefore, mutually dependent. Further, since no cooperation is assumed among the links, we have an instance of the interference channel $[3,4]$ whose complete characterization is still an open problem. Viewed in a noncooperative game theoretic setting [5], the links can be regarded as players whose payoff functions are the individual link rates. Each player is only interested in maximizing the individual rate, without considering its action on the other players. When each player is unilaterally optimal, that is, given the strategies of the other players, a change in the own strategy will not increase the rate, a Nash equilibrium (NE) [6] is reached, and, in general, multiple equilibria are possible. It is of interest to determine these equilibria of decentralized transmission strategies since centralized control causes unnecessary signalling overhead.

A general overview of distributed algorithms for spectrum sharing based on noncooperative game theory can be found in [2]. In [7], an iterative water-filling algorithm (IWFA) for codeword updates is proposed for spectrum allocation in interfering systems. It is shown that the fullspread equilibrium is the only possible outcome of the game under weak interference situations. Such complete spectral overlap is a highly suboptimal solution over a 
wide range of channels. Conditions that guarantee global convergence to such unique NE are presented in [8]. On the other hand, for strong interference channels, it is also shown in [7] that multiple NE corresponding to complete, partial, and no spectral overlap can exist. Further, it is graphically shown that these multiple NEs result in large variations in system performance. Similar game theoretic approaches to codeword adaptation can be found in [9, 10], where stability is analyzed in asynchronous CDMA systems for single and multiple cell wireless systems. Also, noncooperative games for a digital subscriber line (DSL) system have been studied in [11], where an NE is reached when each player maximizes its individual rate in a sequential manner. In [12], it is shown how different operating points, for example, the maximum weighted sum rate, the $\mathrm{NE}$, and the egalitarian solution, can be obtained using an iterative algorithm. However, this scheme requires the transmitters to have different forms of channel state information. An attempt to design noncooperative spectrum sharing rules for decentralized multiuser systems with multiple antennas at both transmitters and receivers can be found in [13]. Also, in [14], a game in which transmitters compete for data rates is presented, and an efficient numerical algorithm to compute the optimal input distribution that maximizes the sum capacity of a multiaccess channel (MAC) is proposed. However, no similar optimal algorithm is known for the general interference channel.

In this paper, we consider a system consisting of two players and study the properties of NE (spectral allocation at equilibrium) obtained by the IWFA. This scenario, albeit simple, allows us to fully characterize the set of achievable operating points and shows that many of the NEs can only be attained under specific initializations. For lowinterference systems, we derive conditions when the fullspread equilibrium is inferior to a separation in signal space and suggest a modification of the IWFA to increase the sum rate. For high-interference systems, we show that the operating points are almost separated in signal space and argue how the convergence properties of the IWFA can be improved. Utilizing global system knowledge, we design a modified game with desirable properties and show how it can be imitated by a decentralized noncooperative scheme corresponding to a modified IWFA. The proposed game is compared to the IWFA by numerical simulations and we illustrate how the results extend, qualitatively, to systems with more players.

The paper is organized as follows. In Section 2, the system model is presented, and the problem is formulated as a noncooperative game. Section 3 provides the analysis for the resulting Nash equilibria and derives the dependencies of the operating points on the various system parameters. An analysis of sum rate is presented in Section 4, and modified games encouraging better system performance are designed in Section 5. The proposed decentralized game is evaluated in Section 6, and finally, conclusions are drawn in Section 7.

Notation: Uppercase boldface letters denote matrices and lowercase boldface letters designate vectors. The superscripts

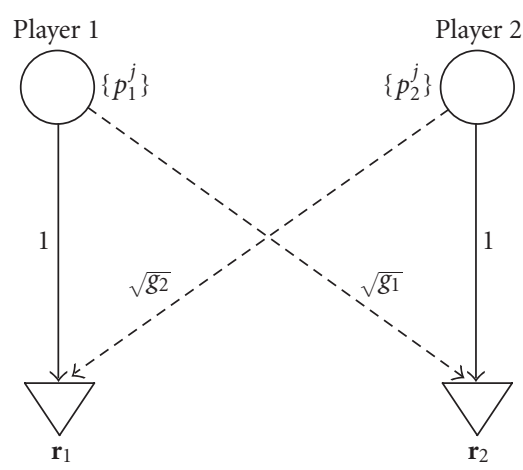

FIGURE 1: System model for two transmit-receive pairs.

$(\cdot)^{T},(\cdot)^{*}$ stand for transposition and Hermitian transposition, respectively. $\mathbf{I}_{N}$ denotes the $N \times N$ identity matrix, and $\underline{1}_{m}$ is the $m \times 1$ vector of ones. Further, let $\operatorname{diag}(\mathbf{x})$ denote a diagonal square matrix whose main diagonal contains the elements of the vector $\mathbf{x}, E[\cdot]$ denotes the expectation operator, and $|\cdot|$ denotes the $l_{1}$-norm.

\section{Problem Formulation and Game Theoretic Approach}

2.1. System Model. We consider a scenario depicted in Figure 1, where two transmit-receive pairs are sharing $N$ orthogonal radio resources, here referred to as subcarriers. Without loss of generality, assume that the system is normalized such that the gain of the transmitted signal is unity at the dedicated receiver. The $N \times 1$ received signal vectors are modeled as

$$
\begin{aligned}
& \mathbf{r}_{1}=\mathbf{s}_{1}+\sqrt{g_{2}} \mathbf{s}_{2}+\mathbf{n}_{1}, \\
& \mathbf{r}_{2}=\sqrt{g_{1}} \mathbf{s}_{1}+\mathbf{s}_{2}+\mathbf{n}_{2},
\end{aligned}
$$

where $\mathbf{r}_{i}$ is the received signal at the $i$ th receiver, and $\mathbf{s}_{i}$ is a complex vector corresponding to transmissions on $N$ subcarriers by the $i$ th transmitter. Further, $g_{i}$ is the cross-gain, and $\mathbf{n}_{i}$ is a zero mean Gaussian noise vector with covariance matrix $E\left[\mathbf{n}_{i} \mathbf{n}_{i}^{*}\right]=\eta_{i} \mathbf{I}_{N}$. To limit the transmit power, each transmitter obeys a long-term power constraint $E\left[\mathbf{s}_{i}^{*} \mathbf{s}_{i}\right]=$ $P_{i}, P_{i}>0, i \in[1,2]$. This system model may represent a multicarrier system with a frequency-flat channel or a time division multiple access (TDMA) system. Though simple, it captures the essence of the spectrum allocation problem and is amenable for a tractable analysis. Such analysis may be useful in devising decentralized spectrum sharing algorithms for more complex scenarios. Similar models have been studied in other works, like $[2,7,8]$.

The individual links can correspond to different instances of the same system or to two different systems. To avoid signaling overhead and retain the dynamic nature of the scenario, we assume that each link does not have information about the parameters used by the other link. Hence, the first player is blind to $P_{2}, \eta_{2}$ and the second player has no information about $P_{1}, \eta_{1}$. Further, since players do not cooperate, the channels $\left\{g_{i}\right\}, i \in[1,2]$ are unknown at either end. 
As we restrict the players to operate as independent units, no interference suppression techniques are devised at the receivers, and the interference is treated as noise. To maximize the mutual information, we model $\boldsymbol{s}_{i}, i \in[1,2]$, as a zero-mean uncorrelated Gaussian vector with covariance matrix $E\left[\mathbf{s}_{i} \mathbf{s}_{i}^{*}\right]=\operatorname{diag}\left(\left\{p_{i}^{j}\right\}_{j=1}^{N}\right), \sum_{j=1}^{N} p_{i}^{j}=P_{i}, p_{i}^{j} \geq 0$, $i \in[1,2]$, where $p_{i}^{j}$ is the power of the $i$ th link for the $j$ th subcarrier. Letting $R_{i}$ denote the rate achieved on link $i$ under Gaussian codebook transmissions, for a given power allocation, we have [15]

$$
\begin{aligned}
& R_{1}=\sum_{j=1}^{N} \log \left(1+\frac{p_{1}^{j}}{g_{2} p_{2}^{j}+\eta_{1}}\right), \\
& R_{2}=\sum_{j=1}^{N} \log \left(1+\frac{p_{2}^{j}}{g_{1} p_{1}^{j}+\eta_{2}}\right) .
\end{aligned}
$$

Note that the individual rates are coupled by the power allocation of both players.

Each player greedily maximizes its individual rate while treating the interference as colored Gaussian noise. Although such selfish behavior may not necessarily lead to improved link rates compared to a cooperative scenario, understanding it allows us to derive various decentralized noncooperative algorithms. These schemes have the advantage of not requiring encoding/decoding by the individual links or using any interference cancellation techniques. Adopting a game theoretical framework provides useful tools to analyze the behavior of greedy systems, and the problem can be tackled in a structured way.

2.2. Game Theoretic Approach to Rate Maximization. The individual rate maximization problem can be cast as a game g

$$
\begin{aligned}
& \underset{\left\{p_{i}^{j}\right\}}{\operatorname{maximize}} R_{i}, \\
& \text { g: } \quad \text { subject to } \sum_{j=1}^{N} p_{i}^{j} \leq P_{i}, \quad p_{i}^{j} \geq 0, \quad \forall i, j,
\end{aligned}
$$

where $\left\{p_{i}^{j}\right\}$ is the set of power allocations $p_{i}^{j}, \forall i, j$. It has been shown in [16] that the outcomes of such noncooperative games are always NE and hence solutions to the set of nonlinear equations highlighting simultaneous water-filling. In particular, $\left\{p_{i}^{j}\right\}$ satisfy

$$
\begin{aligned}
& p_{1}^{j}=\left(\mu_{1}-\left(\eta_{1}+g_{2} p_{2}^{j}\right)\right)^{+}, \\
& p_{2}^{j}=\left(\mu_{2}-\left(\eta_{2}+g_{1} p_{1}^{j}\right)\right)^{+},
\end{aligned}
$$

where $(a)^{+}=\max (0, a)$, and $\mu_{1}, \mu_{2}$ are positive constants such that $\sum_{j=1}^{N} p_{i}^{j}=P_{i}, i \in[1,2]$. These equilibrium points are reached when players update their power using the IWFA in one of the following ways [16].

(1) Sequentially: players update their individual strategies one after the other according to a fixed updating order.
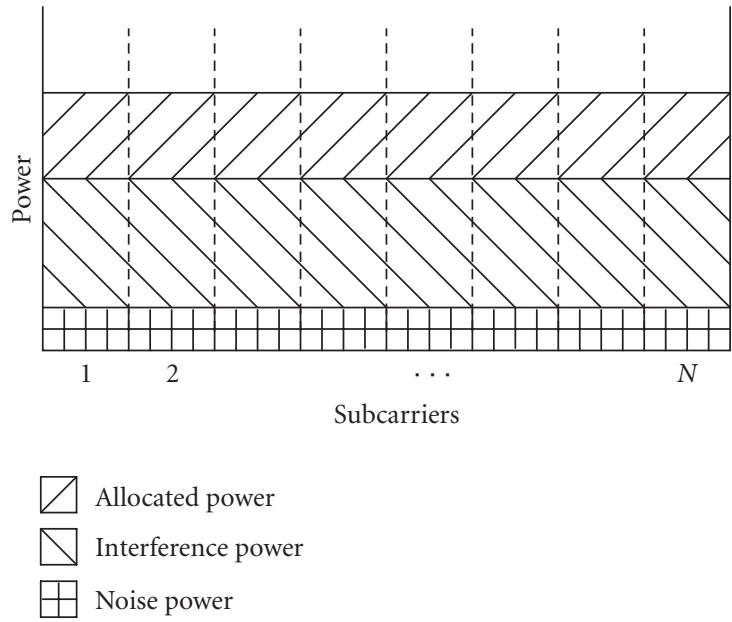

Figure 2: Power allocation corresponding to a complete overlap in signal space, that is, a full-spread equilibrium.
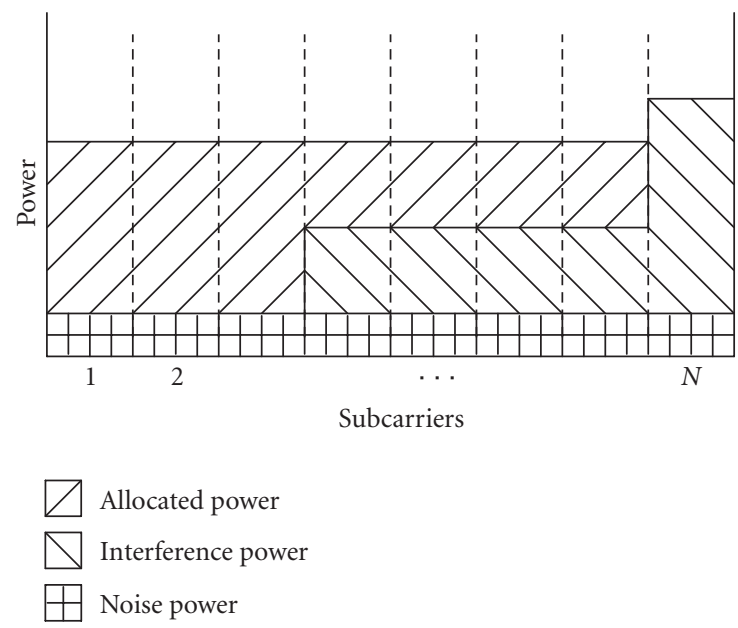

Figure 3: Power allocation corresponding to a partial overlap in signal space.

(2) Simultaneously: at each iteration, all players update their individual strategies simultaneously.

(3) Asynchronously: all players update their individual strategies in an asynchronous way.

For the purpose of tractability, we restrict our analysis to sequential updates.

\section{Properties of Nash Equilibria}

The spectra used by the two players can overlap completely, partially, or be disjoint (completely separated) as illustrated in Figures 2, 3, and 4, respectively. Hence, the resulting power allocation corresponds to one of these scenarios and is likely to depend on the system parameters as well as the particular initialization. In this section, we highlight the dependence of NE on the various system parameters using analytical 

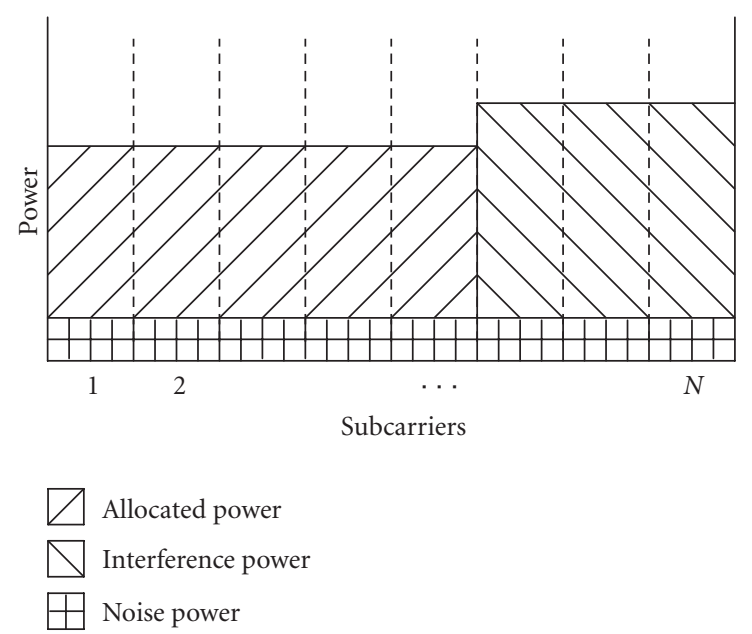

FIgURe 4: Power allocation corresponding to a complete separation in signal space.

methods and derive conditions under which the different power allocations are possible.

3.1. Low-Interference Systems. In communication systems with low interference, individual links generally adapt their operating point to the noise power by neglecting the interference. This is also true for the IWFA when $g_{1} g_{2}<1$. In fact, we have the following.

Theorem 1. When $g_{1} g_{2}<1$, a full-spread equilibrium with $p_{i}^{j}=P_{i} / N, \forall i, j$ is the only possible outcome of the game $g$.

Proof. The proof follows from $[7,17]$ and is omitted for brevity.

Theorem 1 shows that when $g_{1} g_{2}<1$, each player allocates power as if the interfering player was absent, and this behavior is independent of the total power and number of subcarriers employed by the players. However, as we show in later sections, such interference ignorant power allocation may result in suboptimal system performance. To conclude the analysis on the low-interference scenario, we have the following theorem describing the convergence properties of the IWFA.

Theorem 2. When $g_{1} g_{2}<1$, convergence of the IWFA to the full-spread equilibrium is linear with rate $g_{1} g_{2}$.

Proof. See Appendix A.

3.2. High-Interference Systems. When $g_{1} g_{2}>1$, the game admits complete, partial, or no overlap as NE [7]. In the following, we analyze the dynamics of the IWFA and study how these different NEs can be reached. We begin with the full-spread equilibrium.

Theorem 3. When $g_{1} g_{2}>1$, the full-spread equilibrium is an outcome of the IWFA if and only if it is used as an initial point.

\section{Proof. See Appendix B.}

Theorem 3 shows that when $g_{1} g_{2}>1$, players acknowledge the presence of interference and do not occupy all the subcarriers, thereby motivating the term high-interference systems. Since a full-spread equilibrium is only possible under specific initialization, the power allocation at NE generally corresponds to either partial overlap or complete separation in signal space. To study such NE, we denote the subcarrier indices in which the $i$ th player allocates nonzero power by $\mathcal{K}_{i}$ and the set of indices corresponding to partial overlap by $\mathcal{M}=\mathcal{K}_{1} \cap \mathcal{K}_{2}$. Further, let the cardinalities of $\mathcal{K}_{i}$ and $\mathcal{M}$ be $k_{i}$ and $m$, respectively, so that $k_{1}+k_{2}=N+m$. Denoting the complement of $\mathcal{M}$ in $[1, N]$ by $\mathcal{M}^{c}$, we have from [7] that the power allocation at NE satisfies $p_{i}^{j}=$ $c_{i, 1}, \forall j \in \mathcal{K}_{i} \cap \mathcal{M}^{c}$, and $p_{i}^{j}=c_{i, 2}, \forall j \in \mathcal{M}$, where $c_{i, 1}$ and $c_{i, 2}$ are positive constants. Thus, each player allocates equal power at NE for the subcarriers corresponding to a partial overlap. Interestingly, such an initial allocation of power is necessary to achieve a partial overlap and is formalized in the following theorem.

Theorem 4. When $g_{1} g_{2}>1$, IWFA converges to the set of NE, where the power allocations overlap on the subcarrier indices $\mathcal{M}$ only if

(1) $p_{2}^{j}(1)=c_{2}(1), \forall j \in \mathcal{M}$, where $c_{2}(1)$ is a constant;

(2) $k_{j} P_{i}>g_{j}\left(k_{i}-m\right) P_{j}, i \neq j, j \in[1,2]$.

If player 1 initiates the IWFA, one has $p_{1}^{j}(1)=c_{1}(1)$. The $c_{1}(1)$ and $c_{2}(1)$ are chosen such that the total power constraints $P_{1}$ and $P_{2}$ are satisfied.

Proof. See Appendix C.

Hence, we have that partial overlap with $m>1$ can be an outcome of the game only under specific initialization. As an immediate consequence of the results derived in Appendix C, we have the following corollary.

Corollary 1. When condition 2 of Theorem 4 is satisfied for $m=1$, convergence of the IWFA is linear with rate $g_{1} g_{2}\left(k_{1}-\right.$ $1)\left(k_{2}-1\right) / k_{1} k_{2}$.

Since the game $g$ has a nonempty solution set [17], one has that when neither the conditions of Theorems 3 or 4 are satisfied, the resulting operating point must correspond to a complete separation.

These theorems provide useful insight about the structure of the outcomes of the game $g$ and help us to understand the dependence on the various system parameters. However, it is also important to analyze the individual rates of the links. It has been discussed in $[2,8]$ that the NE often is a suboptimal operating point resulting in poor performance for low-interference systems. Therefore, it is important to compare the performance corresponding to the NE with an optimal strategy. The mathematical tractability and fact that complete and partial overlaps are not, in general, solutions provided by the IWFA motivate us to consider the optimal performance under complete separation. 


\section{Analysis of the Sum Rate}

As a global performance measure for the system, we define the sum rate as

$$
R=R_{1}+R_{2}
$$

where $R_{i}$ is the rate achieved on link $i$. For a separated operating point where players 1 and 2 reside in $k$ and $N-k$ signal space dimensions, respectively, the individual rates are

$$
\begin{aligned}
& R_{1}=k \log \left(1+\frac{P_{1}}{k \eta_{1}}\right), \\
& R_{2}=(N-k) \log \left(1+\frac{P_{2}}{(N-k) \eta_{2}}\right) .
\end{aligned}
$$

Here, we explicitly use $k_{1}=k$ and $k_{2}=N-k$ to emphasize the analysis of nonoverlapping power allocations. The optimal signal space partitioning maximizing the sum rate is given by the next theorem.

Theorem 5. The signal space partitioning for player 1 maximizing the sum rate is

$$
k^{o p t}=\frac{P_{1} N \eta_{2}}{P_{1} \eta_{2}+P_{2} \eta_{1}} .
$$

Proof. Note that since $R_{1}$ and $R_{2}$ are concave in $k$, so is the sum rate $R_{1}+R_{2}$. Differentiating the sum rate with respect to $k$ and solving for the roots yield the optimal signal space partitioning $k^{\mathrm{opt}}$.

In general, the optimal partitioning is not an integer and if required, needs to be rounded. Also, since the operating points obtained by the IWFA are NE for the system, not all signal space partitioning are achievable. The following theorem provides the region of all possible signal space partitionings when the IWFA is employed.

Theorem 6. At NE corresponding to a complete separation, the achievable region of signal space dimensions employed by player 1 satisfies

$$
\frac{N}{1+g_{2}\left(P_{2} / P_{1}\right)} \leq k \leq \frac{N}{1+\left(1 / g_{1}\right)\left(P_{2} / P_{1}\right)} .
$$

Proof. Let players 1 and 2 reside in separated signal spaces of dimensions $k$ and $N-k$, respectively, at NE. For player 1 , the allocated power per dimension $P_{1} / k$ satisfies $P_{1} / k \leq$ $g_{2}\left(P_{2} /(N-k)\right)$, since the water level corresponding to the allocated power must be less than the level corresponding to the interference power. Similarly, for player 2, the allocated power $P_{2} /(N-k)$ satisfies $P_{2} /(N-k) \leq g_{1}\left(P_{1} / k\right)$. The region containing the possible signal space partitioning for player 1 is readily obtained combining these expressions.

Note that the region of achievable partitioning is nonempty only when $g_{1} g_{2} \geq 1$ and expands as the channel gains are increased. For $g_{1} g_{2}<1$, this region is empty, and only a full-spread equilibrium is possible. The optimal partitioning needs not to satisfy (8) and conditions can be derived under which the optimal signal space partitioning is a possible outcome of the IWFA.
Theorem 7. The optimal signal space partitioning $k^{o p t}$ is an achievable $N E$ if and only if $g_{1} \geq \eta_{2} / \eta_{1}$ and $g_{2} \geq \eta_{1} / \eta_{2}$.

Proof. Using $g_{1} \geq \eta_{2} / \eta_{1}$ and $g_{2} \geq \eta_{1} / \eta_{2}$ in (8), it is straightforward to see that the optimal signal space partitioning is confined within the region of achievable separations. To prove the only if part, substitute $k$ by $k^{\text {opt }}$ in (8) and simplify.

Theorem 7 enumerates the conditions under which the optimal partitioning is not a possible NE of the game $g$. In such situations, implicit cooperation among the players is necessary to reach the sum rate optimal operation point. This involves the players to follow an etiquette where they do not transmit on a given subcarrier when the other player is employing full power. The following theorem shows when such a strategy results in higher sum rate compared to the IWFA.

Theorem 8. The sum rate corresponding to an operating point with optimal partitioning is higher than or equal to that of the IWFA when

$$
1+\frac{P_{1}}{N \eta_{1}}+\frac{P_{2}}{N \eta_{2}} \geq\left(1-\frac{\eta_{2}}{g_{1} \eta_{1}}\right)\left(1-\frac{\eta_{1}}{g_{2} \eta_{2}}\right) .
$$

Proof. The sum rate for a system where players reside in separated signal spaces of dimension $k$ and $N-k$ is

$$
R_{\text {sep }}=k \log \left(1+\frac{P_{1}}{k \eta_{1}}\right)+(N-k) \log \left(1+\frac{P_{2}}{(N-k) \eta_{2}}\right) .
$$

Using that $P_{1} / k \eta_{1}=P_{2} /(N-k) \eta_{2}$ when $k=k^{\text {opt }}$, we have

$$
R_{\mathrm{sep}}^{\mathrm{opt}}=N \log \left(1+\frac{P_{1}}{N \eta_{1}}+\frac{P_{2}}{N \eta_{2}}\right) .
$$

Further, the sum rate corresponding to a full-spread equilibrium is

$$
R_{\mathrm{fs}}=N \log \left(\left(1+\frac{P_{1}}{g_{2} P_{2}+\eta_{1} N}\right)\left(1+\frac{P_{2}}{g_{1} P_{1}+\eta_{2} N}\right)\right) .
$$

Forming $R_{\mathrm{sep}}^{\mathrm{opt}} \geq R_{\mathrm{fs}}$ yields the desired inequality.

It is clear from Theorem 8 that the sum rate can be increased if the operating point corresponds to the optimal signal space partitioning. However, it follows from [7] that a complete spectral overlap is the only outcome of the IWFA when $g_{1} g_{2}<1$. Unfortunately, the strategy based on Theorem 8 requires information about $\left\{g_{i}\right\},\left\{P_{i}\right\}$, and $\left\{\eta_{i}\right\}, i \in[1,2]$, at each player and also centralized control. This warrants a modification of the IWFA for moving the operating point from a complete spectral overlap to a separation in signal space without requiring any additional system information. The region of achievable partitioning, as defined in Theorems 6 and 7, may contain the optimal separation. However, this depends on the channel gains. By modifying the channel coefficients used in the IWFA, the region can be adjusted to close in on the optimal partitioning. Such modification is equivalent to constructing a new game whose NE has desirable properties. 


\section{Sum Rate Improvements}

As shown in Theorem 8 , the sum rate can be increased by moving to an operating point corresponding to the optimal signal space partitioning. However, such a strategy requires global system knowledge and cooperation among the players making it less attractive from a practical point of view. Using the properties of the NE, we design a game utilizing global system knowledge and show how it can be imitated in a decentralized noncooperative setting.

5.1. Generalized IWFA with Global System Knowledge. When both players have access to global system knowledge, that is, $\left\{P_{i}\right\},\left\{g_{i}\right\}$ and $\left\{\eta_{i}\right\}, i \in[1,2]$, a modified game can be constructed to encourage better operating points compared to those provided by the IWFA. Since a rule-based approach, that switches to another solution for certain parameter values, is extremely tailored to the system model and not easy to generalize to scenarios with more than two players, we utilize the game theoretic framework and show how the individual utility functions of the players can be modified to improve the overall system performance in terms of sum rate.

Using the analysis from Section 4, we can guide the resulting operating point toward the optimal signal space partitioning. As shown in Section 3, the IWFA is generally not globally convergent to the set of NEs with overlap on more than one subcarrier and the region of separated operating points depends highly on the channel gains. Therefore, to direct the operating point toward the optimal signal space partitioning, the interference channel coefficients $g_{1}$ and $g_{2}$ employed by the IWFA should be replaced by the modified gains $\widehat{g}_{1}=c_{1} g_{1}=\eta_{2} / \eta_{1}$ and $\hat{g}_{2}=c_{2} g_{2}=\eta_{1} / \eta_{2}$, where $c_{1}$ and $c_{2}$ are positive scalars. This scaling is done within the algorithm, and the only possible separated operating point will be that corresponding to the optimal partitioning. For a given power allocation, these scaled channel coefficients result in virtual rates as follows:

$$
\begin{aligned}
& \hat{R}_{1}=\sum_{j=1}^{N} \log \left(1+\frac{p_{1}^{j}}{\hat{g}_{2} p_{2}^{j}+\eta_{1}}\right), \\
& \hat{R}_{2}=\sum_{j=1}^{N} \log \left(1+\frac{p_{2}^{j}}{\hat{g}_{1} p_{1}^{j}+\eta_{2}}\right),
\end{aligned}
$$

and a modified game $\hat{g}$ can be formulated as

$$
\begin{array}{ll}
\underset{\left\{p_{i}^{j}\right\}}{\operatorname{maximize}} \hat{\mathrm{R}}_{i}, & \\
\text { subject to } & \sum_{j=1}^{N} p_{i}^{j} \leq P_{i}, \quad p_{i}^{j} \geq 0, \quad \forall i, j .
\end{array}
$$

Using these channel coefficients, the region of separated NE is narrowed to one single point, namely, the optimal partitioning, and from Theorem 4, we know that the resulting operating point will, in general, not overlap on more than one subcarrier. Hence, for a large number of subcarriers, such operating points result in sum rates close to that of the optimal signal space partitioning.
However, we know from Theorem 8 that for $g_{1} g_{2}<1$, the optimal partitioning is not always the best operating point from the sum rate point of view. Since the system parameters are known, both players should determine $k^{\text {opt }}$ and choose the modified game $\hat{g}$ when $R_{\mathrm{fs}}<R_{\mathrm{sep}}^{\mathrm{opt}}$. The resulting sum rate will not be less than that of the IWFA, and the subcarrier allocation will differ in no more than one dimension from the optimal partitioning.

5.2. Generalized IWFA without Global System Knowledge. Since the system parameters might not be available at both players, decentralized games imitating the global game $\hat{g}$ are of high interest. Such a game should encourage separated operating points for $g_{1} g_{2}>1$ and either move away from or move toward the optimal partitioning for $g_{1} g_{2}<1$ depending on the channel strengths. Also, the game should be such that the sum rate is increased as more system parameters become available to the players.

Instead of altering the channel coefficients gains as in the global game $\hat{g}$, we modify the received interference plus noise power employed by the IWFA encouraging the resulting operating point to have desirable characteristics. Letting $I_{i}^{j}$ denote the inverse of the interference plus noise power at link $i$ for subcarrier $j$, we have

$$
\begin{aligned}
& I_{1}^{j}=\left(g_{2} p_{2}^{j}+\eta_{1}\right)^{-1}, \\
& I_{2}^{j}=\left(g_{1} p_{1}^{j}+\eta_{2}\right)^{-1} .
\end{aligned}
$$

Then, we propose to modify the interference plus noise power values for player $i$ into

$$
\tilde{I}_{i}^{j}=M_{i}\left(\frac{I_{i}^{j}}{m\left(\left\{I_{i}\right\}\right)}\right)^{\alpha}, \quad j=1, \ldots, N, i=1,2,
$$

where $\alpha \geq 1$ is a real scalar, $\left\{I_{i}\right\}$ is the set of all $I_{i}^{j}, j=$ $1, \ldots, N, m(\cdot)$ is the arithmetic mean operator, and $M_{i}=$ $\beta m\left(\left\{I_{i}\right\}\right), \beta>0$. The normalization by $m\left(\left\{I_{i}\right\}\right)$ yields a threshold for the decisiveness of the exponent operation, where values above the mean are amplified and others attenuated, while the scaling by $M_{i}$ controls the mean of the modified parameters and implicitly the size of the region of achievable signal space separations. The exponential operation with $\alpha>1$ perturbs a possibly full-spread equilibrium and improves the convergence properties for $g_{1} g_{2}>1$, since separated operating points are encouraged. For a given power allocation, the virtual rate for player $i$ is

$$
\widetilde{R}_{i}=\sum_{j=1}^{N} \log \left(1+\widetilde{I}_{i}^{j} p_{i}^{j}\right)
$$

and the resulting game can be formulated as

$$
\begin{aligned}
& \underset{\left\{p_{i}^{j}\right\}}{\operatorname{maximize}} \widetilde{R}_{i},
\end{aligned}
$$

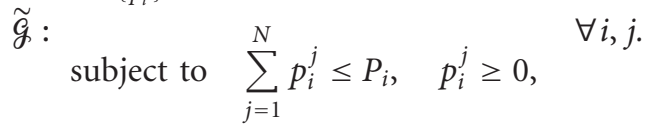


Note, when $\alpha=\beta=1$, this game coincides with the IWFA. As more system information becomes available to the players, the parameters $\alpha$ and $\beta$ can be chosen such that the resulting operating point approaches the optimal partitioning. This can be achieved by altering the range of (8) by a proper choice of the scale factor $\beta$ and affecting the convergence properties with the parameter $\alpha$.

Although we designed the decentralized game for a scenario with two players, such modifications of the interference plus noise power can also be applied to a system with more players as demonstrated in [13] and in a numerical example below.

\section{Numerical Examples}

In this section, we evaluate the system performance in terms of sum rate for the games $q$ and $\widetilde{g}$ and also study their convergence properties.

Each of the values is averaged over 50000 channel and power realizations, and two specific scenarios are considered: $g_{1} g_{2}<1$ and $g_{1} g_{2}>1$. To simplify the exercise, we let $g_{1}$ and $g_{2}$ be uniformly distributed on $[0,1]$ when $g_{1} g_{2}<1$ and identically distributed according to $1+|\mathcal{N}(0,1)|$ when $g_{1} g_{2}>$ 1 . The total power budgets for players 1 and 2 are uniformly distributed on $[0,6]$ and $[0,10]$, respectively, the noise power is 1 , and 10 subcarriers are shared.

The average sum rate for a system whose operating points are given by the games $g$ and $\widetilde{g}$ is shown in Figures 5 and 6 for $g_{1} g_{2}<1$ and $g_{1} g_{2}>1$, respectively. In each of the two interference scenarios, the impact of the scale factor $\beta$ on the average sum rate is depicted for different values of the exponent $\alpha$. Clearly, the modified game $\tilde{g}$ yields a higher average sum rate compared to the IWFA for low-interference systems when $\beta=1$ and $\alpha=2$. Also, from Figure 6, we see that the resulting performance of both games is almost identical for such choice of parameters.

To study the convergence properties, we use the relative change in sum rate as a convergence criterion and set the threshold to $10^{-6}$. For $g_{1} g_{2}<1$ with $\beta=1$ and $\alpha=$ 2 , the modified game $\tilde{g}$ requires 21 iterations on average between the players, whereas the IWFA converges in 17 iterations. This increase is due to the perturbation caused by the exponent operator in (16), where convergence toward a complete overlap is altered. However, for $g_{1} g_{2}>1$, the modified game requires no more than 4 iterations to converge, while 11 iterations are needed for the IWFA. From the properties of NE derived in Section 3, we know that the IWFA will provide an almost separated operating point, and here the exponent operation with $\alpha>1$ encourages the convergence to such a separation.

From the simulation results, we observe that the individual rates at NE corresponding to a partial overlap can be increased by moving the operating point to either complete separation or overlap on one subcarrier. This leads to the conjecture that the IWFA yields Pareto optimal points under arbitrary initialization for high-interference systems.

In order to illustrate how such a decentralized game extends to a scenario with more users, we consider a system

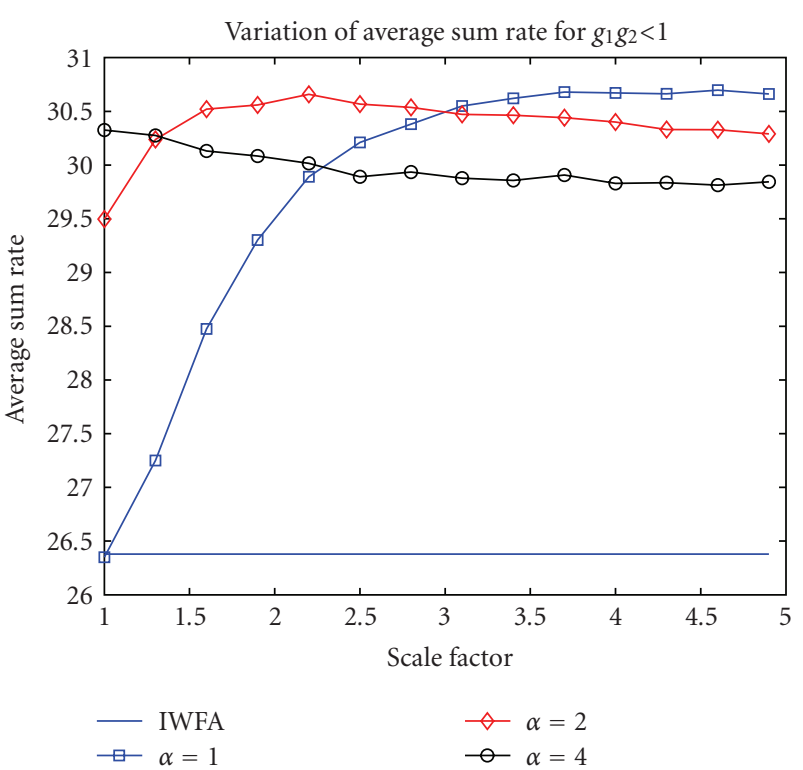

FIGURE 5: A comparison of system performance in terms of sum rate for the decentralized game $\tilde{g}$ and the IWFA when $g_{1} g_{2}<1$. The scale factor $\beta$ is varied between 1 and 5 for $\alpha=1,2$, and 4 .

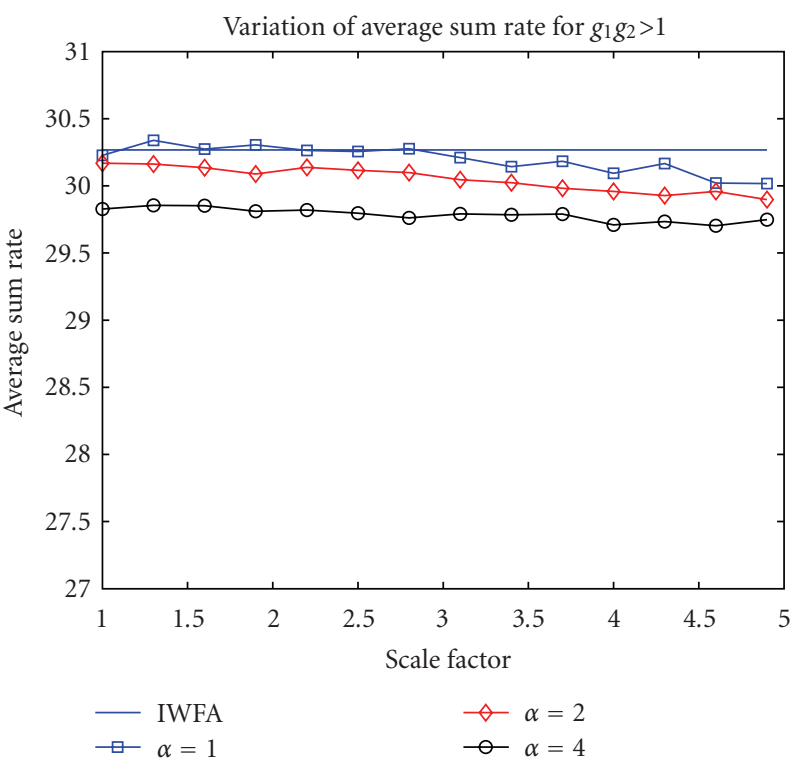

FIGURE 6: A comparison of system performance in terms of sum rate for the decentralized game $\widetilde{g}$ and the IWFA when $g_{1} g_{2}>1$. The scale factor $\beta$ is varied between 1 and 5 for $\alpha=1,2$, and 4 .

consisting of 4 players, whose power budgets are uniformly distributed on $[0,6],[0,8],[0,10]$, and $[0,12]$, respectively. Letting $g_{x y}$ denote the channel gain from transmitter $x$ to receiver $y$, we consider the scenarios when $g_{x y} g_{y x}<1$ and $g_{x y} g_{y x}>1, x \neq y$. When $g_{x y} g_{y x}<1$, the gains are uniformly distributed on $[0,1]$ and identically distributed according to $1+|\mathcal{N}(0,1)|$ when $g_{x y} g_{y x}>1$. Each value is averaged over 50000 channel and power realizations, the noise power is 1 , and 10 subcarriers are shared. 


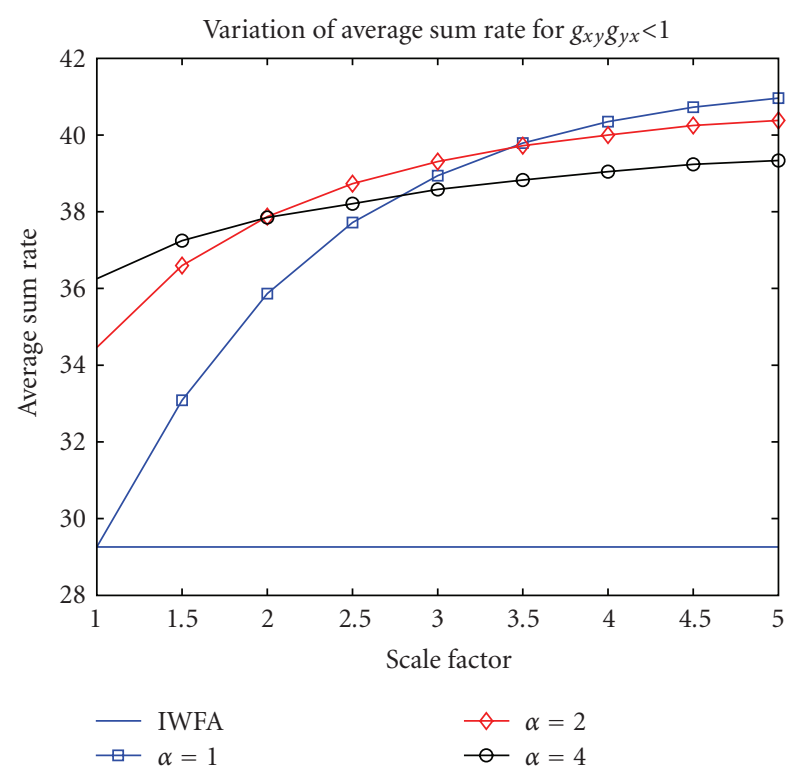

Figure 7: A comparison of system performance in terms of sum rate for the decentralized game $\widetilde{g}$ and the IWFA when $g_{x y} g_{y x}<1$ and 4 players are served. The scale factor $\beta$ is varied between 1 and 5 for $\alpha=1,2$, and 4 .

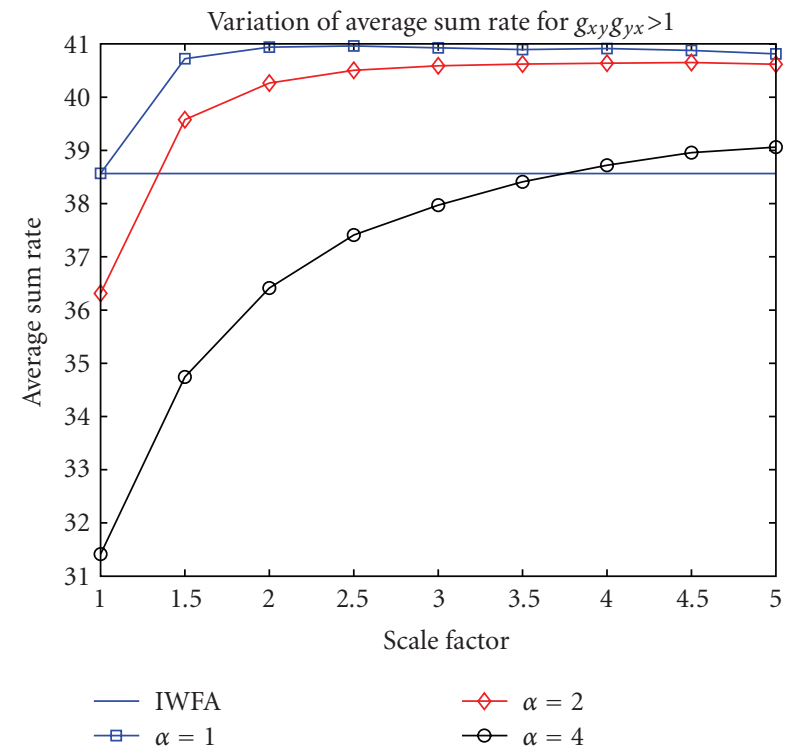

FIGURE 8: A comparison of system performance in terms of sum rate for the decentralized game $\widetilde{g}$ and the IWFA when $g_{x y} g_{y x}>1$ and 4 players are served. The scale factor $\beta$ is varied between 1 and 5 for $\alpha=1,2$, and 4 .

Figures 7 and 8 show the average sum rate for a system whose operating points are given by the games $g$ and $\tilde{g}$ for $g_{x y} g_{y x}<1$ and $g_{x y} g_{y x}>1$, respectively. Similar to the game consisting of two players, the decentralized scheme yields operating points resulting in better system performance compared to the IWFA. In particular, the effect of the perturbation caused by the exponent operation is evident, where separated operating points are encouraged.
Clearly, the overall spectrum utilization benefits from a power allocation with as small overlap between the users as possible.

\section{Conclusion}

In this paper, we have analyzed a decentralized game, where two players compete for available spectrum by greedily maximizing the individual rates and only considering the action of the other player through the experienced interference level. When each player is allocating transmit power using the water-filling algorithm, a Nash equilibrium is reached and, in general, multiple equilibria are possible. We have studied the properties of such NE and characterized the region of achievable operating points. For high-interference systems, these equilibria correspond to almost complete separation in signal space, while for low-interference systems, a fullspread equilibrium is obtained. Further, we showed that the full-spread equilibrium is a stable operating point for the system, but often results in low overall system performance. Therefore, a decentralized algorithm should avoid an initialization with equal power on all subcarriers. We derived the region of achievable signal space partitioning and showed how it depends on the various system parameters. Altering these parameters, we constructed a decentralized noncooperative game whose NE had desirable properties. By properly modifying the value of the interference plus noise power employed by the IWFA, we showed how the overall system performance can be improved. In order to obtain quantitative results, the analysis considered a simple scenario with two links. However, many of the qualitative conclusions will remain also for scenarios with more players.

\section{Appendices}

\section{A. Proof of Theorem 2}

Without loss of generality, let the IWFA be initiated by player 2. Further, let $p_{i}^{j}(n)$ denote the power allocation of the $i$ th link for the $j$ th subcarrier during the $n$th iteration, and let $\mathcal{N}_{i, n}$ be the set containing the subcarrier indices for which link $i$ allocates nonzero power during the $n$th iteration. Then, water-filling yields

$$
\begin{aligned}
& p_{2}^{j}(n)=-g_{1} p_{1}^{j}(n-1)+\frac{1}{r_{2, n}}\left(P_{2}+\sum_{l \in \mathcal{N}_{2, n}} g_{1} p_{1}^{l}(n-1)\right), \\
& p_{1}^{j}(n)=-g_{2} p_{2}^{j}(n)+\frac{1}{r_{1, n}}\left(P_{1}+\sum_{l \in \mathcal{N}_{1, n}} g_{2} p_{2}^{l}(n)\right),
\end{aligned}
$$

where $r_{i, n}$ denotes the cardinality of $\mathcal{N}_{i, n}$. Since the outcome of the IWFA is the full-spread equilibrium, there exists a finite $n_{0}$ such that $p_{i}^{j}(n)>0, \forall n \geq n_{0}, \forall j$ and $i \in[1,2]$.

We start by showing that the IWFA cannot converge in $n_{0}$ (finite) iterations under random initialization [18]. Note that the equilibrium is reached at $n_{0}=1$ only if the algorithm 
is initialized with the operating point corresponding to a complete spectral overlap. Assume that the NE is reached for $n_{0}>1$. Then, $p_{2}^{j}\left(n_{0}+1\right)=p_{2}^{j}\left(n_{0}\right)$ and $r_{2, n}=N, \forall n \geq n_{0}$. Using that $\sum_{j} p_{1}^{j}(n)=P_{1},($ A. 1$)$ yields $p_{1}^{j}\left(n_{0}-1\right)=p_{1}^{j}\left(n_{0}\right)$. This implies $r_{1, n_{0}-1}=N$, and (A.2) yields $p_{2}^{j}\left(n_{0}-1\right)=$ $p_{2}^{j}\left(n_{0}\right)$. By recursion, we see that $p_{i}^{j}(n)$ is constant for all $n \leq n_{0}$. Hence, equilibrium is reached at a finite $n_{0}$ only when the IWFA is initialized with this point.

Since $r_{1, n}=r_{2, n}=N, \forall n \geq n_{0}$, (A.1) and (A.2) yield

$$
\begin{aligned}
& p_{1}^{j}(n+1)-p_{1}^{j}(n)=g_{1} g_{2}\left(p_{1}^{j}(n)-p_{1}^{j}(n-1)\right), \\
& p_{2}^{j}(n+1)-p_{2}^{j}(n)=g_{1} g_{2}\left(p_{2}^{j}(n)-p_{2}^{j}(n-1)\right) .
\end{aligned}
$$

It follows from (A.3) that the convergence of the IWFA is linear with rate $g_{1} g_{2}$.

\section{B. Proof of Theorem 3}

Assuming that the full-spread equilibrium is the outcome of the game $g$, it follows from Appendix A that the IWFA cannot converge in $n_{0}$ iterations under random initialization [18]. Further, (A.3) hold for $n \geq n_{0}$. We now show that a fullspread equilibrium is not attained for $n>n_{0}$. By the Cauchy criterion, $p_{i}^{j}(n)$ converges if and only if $\left|p_{i}^{j}(n+1)-p_{i}^{j}(n)\right|$ converges to 0 as $n \rightarrow \infty$. However, since $g_{1} g_{2}>1$, it is clear from (A.3) that $\left|p_{i}^{j}(n+1)-p_{i}^{j}(n)\right|$ cannot converge to 0 , unless $p_{i}^{j}\left(n_{0}+1\right)-p_{i}^{j}\left(n_{0}\right)=0, \forall j$. From Appendix A, we see that such a scenario is not possible for a random initialization, thereby proving the theorem.

\section{Proof of Theorem 4}

Assuming partial overlap at convergence, there exists a finite $n_{0}$ such that $p_{i}^{j}(n)>0, \forall n \geq n_{0}, j \in \mathcal{K}_{i}, i \in[1,2]$. The following lemma is necessary to prove the theorem.

Lemma 1. Defining $n_{0}$ as above, one has $p_{i}^{j}(\hat{n})>0, \forall j \in \mathcal{M}$, $i \in[1,2]$ and $1<\hat{n} \leq n_{0}$.

Proof. If, for some $\hat{n}<n_{0}$, we have $p_{1}^{j}(\hat{n})=0, j \in \mathcal{M}$, then $p_{2}^{j}(n), j \in \mathcal{M}, n \geq \hat{n}$ has the largest value among all $j \in[1, N]$. However, $p_{2}^{j}\left(n_{0}\right)$ has the largest value for all $j \in \mathcal{M}^{c} \cap \mathcal{K}_{2}$ as it does not experience any interference. This leads to a contradiction and thereby proves the lemma for $i=1$. Similar arguments hold for $i=2$.

To simplify the analysis, we consider two cases: (1) $k_{i}>$ $m, \forall i$ and (2) $k_{i}=m$ for some $i$.

Case $1\left(k_{i}>m\right)$. Stack the powers corresponding to the subcarriers with spectral overlap in the vector $\underline{p}_{i}(n)=$ $\left[\left\{p_{i}^{j}(n)\right\}_{j \in \mathcal{M}}\right]^{T}, i \in[1,2]$, and denote the difference in power for two consecutive updates by $\underline{\delta}^{i}(n)=\underline{p}_{i}(n)-$ $\underline{p}_{i}(n-1), i \in[1,2]$. Then, for $n \geq n_{0}$, we can write (A.1) and (A.2) as

$$
\begin{aligned}
& \underline{p}_{2}(n)=g_{1} \mathbf{M}_{2} \underline{p}_{1}(n-1)+\frac{P_{2}}{k_{2}} \underline{1}_{m}, \\
& \underline{p}_{1}(n)=g_{2} \mathbf{M}_{1} \underline{p}_{2}(n)+\frac{P_{1}}{k_{1}} \underline{1}_{m},
\end{aligned}
$$

where $\mathbf{M}_{i}=-\mathbf{I}_{m}+\left(1 / k_{i}\right) \underline{1}_{m} \underline{1}_{m}^{T}, i \in[1,2], \mathbf{I}_{m}$ is an $m \times m$ identity matrix, and $\underline{1}_{m}$ is an $m \times 1$ vector of ones. The following properties of $\mathbf{M}_{i}$ are useful in the subsequent steps.

(i) $\mathbf{M}_{i}$ is Hermitian with eigenvalue -1 with multiplicity $m-1$ and $\left(-1+m / k_{i}\right)$ with multiplicity 1 . Further, when $k_{i}>m$, all eigenvalues of $\mathbf{M}_{i}$ are nonzero. Thus, $\mathbf{M}_{i}$ is invertible for $k_{i}>m$.

(ii) The eigenvector corresponding to the eigenvalue $\left(-1+m / k_{i}\right)$ is $\underline{1}_{m}$ and is orthogonal to the eigenvectors corresponding to the eigenvalue -1 . Since the eigenvectors of $\mathbf{M}_{1}$ and $\mathbf{M}_{2}$ are identical, they commute [19]. Further, the matrix $\mathbf{M}_{i_{1}} \mathbf{M}_{i_{2}}, i_{1}, i_{2} \in$ $[1,2]$ has eigenvalue 1 with multiplicity $m-1$ and $\left(-1+m / k_{i_{1}}\right)\left(-1+m / k_{i_{2}}\right)$ with multiplicity 1 . Thus, $\mathbf{M}_{i_{1}} \mathbf{M}_{i_{2}}$ is invertible for $k_{i_{l}}>m, l \in[1,2]$.

We first show that an appropriate initialization satisfying $p_{2}^{j}(1)=c_{2}(1), \forall j \in \mathcal{M}$ is necessary for the IWFA to converge in $n_{0}$ (finite) iterations. Assuming an equilibrium at $n=n_{0}$, it follows from [7] that $p_{i}^{j}\left(n_{0}\right)=c_{i}\left(n_{0}\right), \forall j \in$ $\mathcal{M}, i \in[1,2]$. Evaluating (A.1) for $n=n_{0}$ and $n=n_{0}+1$ and noting that $\underline{p}_{2}\left(n_{0}\right)=\underline{p}_{2}\left(n_{0}+1\right)$, we have $\underline{p}_{1}\left(n_{0}-\right.$ $1)=\underline{p}_{1}\left(n_{0}\right)=c_{1}\left(n_{0}\right) \underline{1}_{m}$ (this can also be argued using (C.1) and the invertibility properties of $\mathbf{M}_{2}$ ). Otherwise there exists an index $j$ such that $p_{2}^{j}\left(n_{0}-1\right)=p_{2}^{j}\left(n_{0}\right)=0$, which is not possible using water-filling. Then, we have that $p_{2}^{j}\left(n_{0}-1\right)=c_{2}\left(n_{0}-1\right), j \in \mathcal{M}$, that is, $p_{2}^{j}\left(n_{0}-1\right)$ is constant for $j \in \mathcal{M}$. Applying this repeatedly yields equal power allocation for $p_{2}^{j}(1), j \in \mathcal{M}$, if $p_{i}^{j}(\hat{n}) \neq 0$ for $j \in \mathcal{M}$ and all $\hat{n}<n_{0}$. Lemma 1 eliminates such a possibility and, therefore, equilibrium can be reached in $n_{0}$ iterations only under specific initialization.

Using (C.1) and (C.2), for all $n \geq n_{0}$, we have

$$
\begin{aligned}
& \underline{\delta}^{2}(n+1)=g_{1} \mathbf{M}_{2} \underline{\delta}^{1}(n), \\
& \underline{\delta}^{1}(n+1)=g_{2} \mathbf{M}_{1} \underline{\delta}^{2}(n+1) .
\end{aligned}
$$

Further, substituting (C.3) in (C.4) and vice versa, we obtain

$$
\begin{array}{ll}
\underline{\delta}^{2}(n)=g_{1} g_{2} \mathbf{M}_{2} \mathbf{M}_{1} \underline{\delta}^{2}(n-1), & n \geq n_{0}+2, \\
\underline{\delta}^{1}(n)=g_{1} g_{2} \mathbf{M}_{1} \mathbf{M}_{2} \underline{\delta}^{1}(n-1), & n \geq n_{0}+1 .
\end{array}
$$

Let $\mathbf{M}_{i}=\mathbf{V} \boldsymbol{\Lambda}_{i} \mathbf{V}^{*}$ be the eigenvalue decomposition of $\mathbf{M}_{i}$ and $\underline{\phi}_{n}^{i}=\mathbf{V}^{*} \underline{\delta}^{i}(n)$. Then, (C.5) can be written as

$$
\underline{\phi}_{n}^{i}=g_{1} g_{2} \Lambda \underline{\phi}_{n-1}^{i}, \quad i \in[1,2]
$$

where $\boldsymbol{\Lambda}=\operatorname{diag}\left(1,1, \ldots, 1,\left(k_{1}-m\right)\left(k_{2}-m\right) / k_{1} k_{2}\right)$. Equations (C.5) and (C.6) suggest that the IWFA converges if and only 
if $\underline{\phi}_{n}^{i}$ converges to a vector with all components equal to zero. Using $\Lambda$, we have that $\underline{\phi}_{n}^{i} \rightarrow \underline{0}$ only if

$$
\begin{gathered}
\underline{\phi}_{n_{0}}^{i}(k)=0, \quad k \in[1, m-1], \\
\frac{\left(k_{1}-m\right)\left(k_{2}-m\right)}{k_{1} k_{2}}<\frac{1}{g_{1} g_{2}},
\end{gathered}
$$

where we used (C.4) and (C.6) to show that $\underline{\phi}_{n_{0}}^{i}(k)=0$ implies $\underline{\phi}_{n}^{i}(k)=0, \forall n>n_{0}+1$. Thus, (C.7) shows that partial overlap is an outcome of the game $g$ only under judicious initialization. Further, (C.8) gives a condition on system parameters for convergence.

We now explore condition (C.7) in more detail. Combining (C.1) and (C.2), we get

$$
\begin{array}{r}
\underline{p}_{1}(n)=g_{1} g_{2} \mathbf{M}_{1} \mathbf{M}_{2} \underline{p}_{1}(n-1)+\left(-1+m / k_{1}\right) g_{2} \frac{P_{2}}{k_{2}} \underline{1}_{m}+\frac{P_{1}}{k_{1}} \underline{1}_{m}, \\
\forall n \geq n_{0} .
\end{array}
$$

Recall that the eigenvector matrix of $\mathbf{M}_{i}$ has the form $\mathbf{V}=$ $\left[\mathbf{Q},(1 / \sqrt{m}) \underline{1}_{m}\right]$, with $\mathbf{Q}^{*} \mathbf{M}_{1} \mathbf{M}_{2}=\mathbf{Q}^{*}$ and $\mathbf{Q}^{*} \underline{1}_{m}=0$. Using this in (C.9) yields

$$
\mathbf{Q}^{*} \underline{p}_{1}(n)=g_{1} g_{2} \mathbf{Q}^{*} \underline{p}_{1}(n-1), \quad \forall n \geq n_{0} .
$$

We then have $\underline{\phi}_{n_{0}}^{1}(k)=0, k \in[1, m-1]$, if and only if $\mathbf{Q}^{*} \underline{\delta}^{1}\left(n_{0}\right)=\underline{0}$. Further, from (C.10), we have $\mathbf{Q}^{*} \underline{\delta}^{1}\left(n_{0}\right)=$ $\mathbf{Q}^{*} \underline{p}_{1}\left(n_{0}\right)-\mathbf{Q}^{*} \underline{p}_{1}\left(n_{0}-1\right)=\left(g_{1} g_{2}-1\right) \mathbf{Q}^{*} \underline{p}_{1}\left(n_{0}-1\right)$. Thus, $\mathbf{Q}^{*} \underline{\delta}^{1}\left(n_{0}\right)=\underline{0}$ implies $\mathbf{Q}^{*} \underline{p}_{1}\left(n_{0}-1\right)=\underline{0}$ as $g_{1} g_{2}>1$. Hence, $\mathbf{Q}^{*} \underline{p}_{1}\left(n_{0}-1\right)=\underline{0}$ and $p_{1}^{j}\left(n_{0}-1\right)$ is constant for all $j \in \mathcal{M}$. As in the discussion preceding (C.3), it can be shown that (C.7) holds only under specific initialization. Hence, condition (1) of Theorem 4 is shown.

To show (C.8), let $p_{i}^{j}=p_{i}^{\text {ol }}, j \in \mathcal{M}$ and $p_{i}^{j}=p_{i}^{\text {nol }}, j \in$ $\mathcal{K}_{i} \cap \mathcal{M}^{c}$ denote the power levels of player $i$ for the subcarriers with and without spectral overlap, respectively. Then, for player 1 , we have

$$
\begin{gathered}
\left(k_{1}-m\right) p_{1}^{\mathrm{nol}}+m p_{1}^{\mathrm{ol}}=P_{1}, \\
p_{1}^{\mathrm{ol}}+g_{2} p_{2}^{\mathrm{ol}}=p_{1}^{\mathrm{nol}},
\end{gathered}
$$

where (C.11) follows from the power constraint of player 1 and (C.12) is due to the water-filling. Similarly, for player 2, we have

$$
\begin{gathered}
\left(k_{2}-m\right) p_{2}^{\mathrm{nol}}+m p_{2}^{\mathrm{ol}}=P_{2}, \\
p_{2}^{\mathrm{ol}}+g_{1} p_{1}^{\mathrm{ol}}=p_{2}^{\mathrm{nol}} .
\end{gathered}
$$

Solving these equations for $p_{1}^{\mathrm{ol}}$ and $p_{2}^{\mathrm{ol}}$, we get

$$
\begin{aligned}
& p_{1}^{\mathrm{ol}}=\frac{k_{2} P_{1}-g_{2}\left(k_{1}-m\right) P_{2}}{k_{1} k_{2}-g_{1} g_{2}\left(k_{1}-m\right)\left(k_{2}-m\right)}, \\
& p_{2}^{\mathrm{ol}}=\frac{k_{1} P_{2}-g_{1}\left(k_{2}-m\right) P_{1}}{k_{1} k_{2}-g_{1} g_{2}\left(k_{1}-m\right)\left(k_{2}-m\right)} .
\end{aligned}
$$

From (C.8), we have that the denominator is positive and, therefore, the overlapping power allocations are nonzero only when $k_{1} P_{2}>g_{1}\left(k_{2}-m\right) P_{1}$ and $k_{2} P_{1}>g_{2}\left(k_{1}-m\right) P_{2}$.

Case $2\left(k_{i}=m\right.$ for some $\left.i\right)$. As in the earlier case, it can be shown that the IWFA converges in $n_{0}$ iterations only under specific initialization. For random initialization, it can be shown that

$$
\begin{aligned}
& p_{2}^{j}(n+1)-p_{2}^{j}(n)=-g_{1}\left(p_{1}^{j}(n)-p_{1}^{j}(n-1)\right), \quad \forall j \in \mathcal{M}, \\
& p_{1}^{j}(n+1)-p_{1}^{j}(n)=-g_{2}\left(p_{2}^{j}(n+1)-p_{2}^{j}(n)\right), \quad \forall j \in \mathcal{M},
\end{aligned}
$$

when $k_{i}=m$ for some $i$. Then, it immediately follows that an equilibrium is not reached for $g_{1} g_{2}>1$.

\section{Acknowledgments}

This work is supported in part by the FP6 project Cooperative and Opportunistic Communications in Wireless Networks (COOPCOM), Project no. FP6-033533. Part of the material was presented at the Asilomar Conference on Signals, Systems, and Computers 2008 and the Global Communications Conference 2008.

\section{References}

[1] A. MacKenzie and L. DaSilva, Game Theory for Wireless Engineers, Morgan \& Claypool, San Rafael, Calif, USA, 2006.

[2] R. Etkin, A. Parekh, and D. Tse, "Spectrum sharing for unlicensed bands," IEEE Journal on Selected Areas in Communications, vol. 25, no. 3, pp. 517-528, 2007.

[3] R. Ahlswede, "The capacity region of a channel with two senders and two receivers," Annals of Probability, vol. 2, no. 5, pp. 805-814, 1974.

[4] A. B. Carleial, "Interference channels," IEEE Transactions on Information Theory, vol. 24, no. 1, pp. 60-70, 1978.

[5] M. J. Osborne and A. Rubinstein, A Course in Game Theory, MIT Press, Cambridge, Mass, USA, 1994.

[6] J. F. Nash Jr., "Equilibrium points in n-person games," Proceedings of the National Academy of Sciences of the United States of America, vol. 36, no. 1, pp. 48-49, 1950.

[7] O. Popescu, Interference avoidance for wireless systems with multiple receivers, Ph.D thesis, Rutgers University, New Brunswick, NJ, USA, 2004.

[8] G. Scutari, D. P. Palomar, and S. Barbarossa, "Optimal linear precoding strategies for wideband non-cooperative systems based on game theory-part II: algorithms," IEEE Transactions on Signal Processing, vol. 56, no. 3, pp. 1250-1267, 2008.

[9] J. E. Hicks, A. B. MacKenzie, J. A. Neel, and J. H. Reed, "A game theory perspective on interference avoidance," in Proceedings of IEEE Global Telecommunications Conference (GLOBECOM '04), vol. 1, pp. 257-261, Dallas, Tex, USA, November-December 2004.

[10] C. W. Sung and K. K. Leung, "On the stability of distributed sequence adaptation for cellular asynchronous DS-CDMA systems," IEEE Transactions on Information Theory, vol. 49, no. 7, pp. 1828-1831, 2003.

[11] W. Yu, G. Ginis, and J. M. Cioffi, "Distributed multiuser power control for digital subscriber lines," IEEE Journal on Selected Areas in Communications, vol. 20, no. 5, pp. 1105-1115, 2002. 
[12] E. A. Jorswieck and E. G. Larsson, “THE MISO interference channel from a game-theoretic perspective: a combination of selfishness and altruism achieves Pareto optimality," in Proceedings of IEEE International Conference on Acoustics, Speech and Signal Processing (ICASSP '08), pp. 5364-5367, Las Vegas, Nev, USA, April 2008.

[13] P. von Wrycza, M. Bengtsson, and B. Ottersten, "Decentralized dynamic channel allocation for MIMO systems," in Proceedings of the 41st Asilomar Conference on Signals, Systems and Computers (ACSSC '07), pp. 1689-1693, Pacific Grove, Calif, USA, November 2007.

[14] W. Yu, W. Rhee, S. Boyd, and J. M. Cioffi, "Iterative waterfilling for Gaussian vector multiple-access channels," IEEE Transactions on Information Theory, vol. 50, no. 1, pp. 145152, 2004.

[15] T. M. Cover and J. A. Thomas, Elements of Information Theory, John Wiley \& Sons, New York, NY, USA, 1991.

[16] G. Scutari, D. P. Palomar, and S. Barbarossa, "Asynchronous iterative water-filling for Gaussian frequency-selective interference channels," IEEE Transactions on Information Theory, vol. 54, no. 7, pp. 2868-2878, 2008.

[17] G. Scutari, D. P. Palomar, and S. Barbarossa, "Optimal linear precoding strategies for wideband noncooperative systems based on game theory-part I: Nash equilibria," IEEE Transactions on Signal Processing, vol. 56, no. 3, pp. 1230-1249, 2008.

[18] D. P. Bertsekas and J. N. Tsitsiklis, "Parallel distributed computation: numerical methods," Nonlinear Programming, Athena Scientific, Belmont, MA, USA, 1997.

[19] R. A. Horn and C. R. Johnson, Matrix Analysis, Cambridge University Press, Cambridge, UK, 1985. 